\title{
LLLT e avulsão dentária: implicações nas propedêuticas endodônticas e periodônticas
}

LLLT and tooth avulsion: implications of LLLT in endodontic and periodontic procedures LLLT y avulsión dental: implicaciones de LLLT en procedimientos de endodoncia y periodoncia Larissa dos Santos DE MORAES ${ }^{1}$ Christine Men MARTINS ${ }^{2}$

${ }^{1}$ Curso de Graduação em Odontologia, Universidade do Oeste Paulista (UNOESTE) 19050-920, Presidente Prudente, Sao Paulo, Brasil ${ }^{2}$ Professora Titular, Disciplinas de Clínica Odontológica e Endodontia, Curso de Graduação em Odontologia, Universidade do Oeste Paulista (UNOESTE) 19050-920, Presidente Prudente, Sao Paulo, Brasil

\section{Resumo}

Introdução: Um dos principais traumatismos dentários com graves consequências que acomete a população é a avulsão dentária, caracterizada pela completa exarticulação do dente para fora do seu alvéolo. Como consequência desse trauma, as fibras do ligamento periodontal, cemento e polpa são agredidas e pode ocorrer a reabsorção radicular. Devido à eficácia do uso da laserterapia de baixa potência (low level laser therapy - LLLT) em tratamentos periodontais e endodônticos, seu uso parece promissor em casos de avulsão dentária, podendo aumentar as taxas de sucesso. Objetivo: Realizar uma revisão de literatura para compreender a ação do LLLT sobre as propedêuticas endodônticas e periodonticas; apresentar o conhecimento atual e científico sobre a eficácia da LLLT na avulsão dentária; e observar falhas de pesquisas sobre o tema. Revisão de literatura: O LLLT reduz a dor pós-operatória na terapia e retratamento do canal radicular, tendo uma diminuição significativamente maior em relação aos anti-inflamatórios. Na periodontia há a redução de bactérias e cálculos, neutralização de endotoxina e diminuição de profundidade de bolsas. Poucos estudos avaliaram sua aplicação em tratamentos para reimplante dentário, os quais mostraram uma melhora a cicatrização, aumento na espessura da camada de dentina reacional e diminuição da inflamação. Considerações finais: A irradiação do LLLT promove reações bioquímicas, originando a estimulação do reparo tecidual, regeneração e redução dos processos inflamatórios, podendo ser um tratamento promissor para casos de avulsão e reimplante dentários. Mais pesquisas devem ser realizadas.

Descritores: Terapia com Luz de Baixa Intensidade; Avulsão Dentária; Reimplante Dentário; Endodontia; Periodontia.

\section{Abstract}

Introduction: One of the major dental trauma that affects the population which leads to important consequences is tooth avulsion, characterized by complete exarticulation of the tooth out of its socket. As consequence of this trauma, periodontal ligament fibers, cementum and pulp are attacked and root resorption can occur. Due to the effectiveness of using low level laser therapy (LLLT) in periodontal and endodontic treatments, its use seems promising in cases of dental avulsion, which may increase success rates. Objective: Comprehensively review the literature to understand the LLLT's action on endodontic and periodontic treatments; present current and scientific knowledge about the effectiveness of LLLT in dental avulsion; and observe research failures on the topic. Literature review: LLLT reduces postoperative pain in primary and retreatment of the root canal, with a significantly greater decrease compared to anti-inflammatory drugs. In periodontics there is a reduction in bacteria and stones, neutralization of endotoxin and a decrease in the depth of bags. Few studies have evaluated its application in treatments for dental replantation. However, current literature demonstrated an improvement in healing, an increase in the thickness of the reactive dentin layer and a decrease in inflammation. Final considerations: LLLT irradiation promotes biochemical reactions, resulting in the stimulation of tissue repair, regeneration and reduction of inflammatory processes, which can be a promising treatment for cases of avulsion and dental replantation. Further researchers should be performed.

Descriptors: Low-Level Light Therapy; Tooth Avulsion; Tooth Replantation; Endodontics; Periodontics.

\section{Resumen}

Introducción: Uno de los principales traumas dentales con graves consecuencias que afecta a la población es la avulsión dental, caracterizada por la exarticulación completa del diente fuera de su cavidad. Como consecuencia de este trauma, las fibras del ligamento periodontal, cemento y pulpa son atacadas y puede producirse la reabsorción de la raíz. Debido a la efectividad del uso de la terapia con láser de bajo nivel (LLLT) en tratamientos periodontales y endodónticos, su uso parece prometedor en casos de avulsión dental, lo que puede aumentar las tasas de éxito. Objetivo: realizar una revisión de la literatura para comprender la acción del LLLT sobre la propedéutica endodóntica y periodontica; presentar conocimiento actual y científico sobre la efectividad de LLLT en avulsión dental; y observar fallas de investigación sobre el tema. Revisión de literatura: LLLT reduce el dolor postoperatorio en la terapia y el retratamiento del conducto radicular, con una disminución significativamente mayor en comparación con los medicamentos antiinflamatorios. En periodoncia hay una reducción en bacterias y cálculos, neutralización de endotoxinas y una disminución en la profundidad de las bolsas. Pocos estudios han evaluado su aplicación en tratamientos para la reimplantación dental, que mostraron una mejora en la curación, un aumento en el grosor de la capa de dentina reactiva y una disminución de la inflamación. Consideraciones finales: la irradiación LLLT promueve reacciones bioquímicas, lo que resulta en la estimulación de la reparación de tejidos, la regeneración y la reducción de procesos inflamatorios, lo que puede ser un tratamiento prometedor para casos de avulsión y reimplantación dental. Se debe realizar más investigación.

Descriptores: Terapia por Luz de Baja Intensidad; Avulsión de Diente; Reimplante Dental; Endodoncia; Periodoncia.

INTRODUÇÃO

$$
\text { O traumatismo dentário ocorre }
$$

frequentemente em crianças que estão na préescola e em idade escolar, mas engloba somente $5 \%$ das lesões em adultos ${ }^{1}$. Segundo Diangelis e colaboradores ${ }^{1}, 33 \%$ dos jovens adultos já sofreram trauma da dentição permanente (antes dos 19 anos) e $25 \%$ de todas as crianças em idade escolar experimentaram um trauma dentário. Destarte, as lesões dentárias traumáticas mais decorrentes na dentição decídua são as lesões de luxação e na dentição permanente as fraturas coronárias são mais predominantes ${ }^{1}$.

As lesões traumáticas podem ter várias etiologias, a saber: prática de esportes violentos, acidentes de bicicleta, acidentes de trânsito, violência física, durante um procedimento de intubação e durante a anestesia geral ${ }^{2,3}$. Pelo fato dos homens se envolverem mais em esportes, brigas e 
acidentes de carro, eles são duas vezes mais susceptíveis ao traumatismo dentário do que indivíduos do gênero feminino ${ }^{3}$. Corroborando, durante a infância os meninos sofrem mais com lesões de tecido duro e de polpa se comparado às meninas ${ }^{3}$.

Os dentes decíduos apresentam mais comumente os diferentes tipos de lesões de luxação, como subluxação $(38,6 \%)$, luxação oral $(22,5 \%)$ e avulsão (16,6\%), já os dentes permanentes há a predominância de fraturas de esmalte e dentina $(32 \%)^{4-6}$. Os traumatismos dentários podem levar ou não complicações ou fraturas de coroa e/ou raiz, podendo estar unidas a uma necrose pulpar. As consequências em casos de avulsão ou intrusão podem ser mais perigosas, como uma reabsorção de raiz externa ${ }^{3}$.

A avulsão dentária, que consiste na completa exarticulação do dente para fora da cavidade alveolar, é uma das lesões dentárias mais graves e o prognóstico é consequência das ações tomadas imediatamente após a avulsão $^{7-9}$. A avulsão lesa as fibras radiculares que tem por função unir os dentes à mandíbula e o suprimento sanguíneo - que penetram através do canal radicular ${ }^{10}$. $O$ tratamento ideal para a avulsão dentária é o reposicionamento do dente em sua cavidade, que deve ser realizada o quanto antes juntamente com vistas à prevenção dos tecidos periodontais ${ }^{11,12}$.

Com a execução adequada do reimplante do dente avulsionado, evita-se uma inflamação, anquilose e até mesmo uma reabsorção radicular ${ }^{13}$. Ademais, a avulsão em dentes decíduos tem o risco de distúrbio de desenvolvimento nos dentes permanentes, que é maior quanto menor a idade da criança no momento da lesão ${ }^{14}$. Logo, conclui-se que há um comprometimento da qualidade de vida nos pacientes que sofreram com a avulsão dentária e que as diferentes opções de tratamento influenciam, sendo sempre a melhor escolha o replantio imediato do dente ${ }^{15}$.

A ciência busca descobrir tratamentos que aumentem as taxas de sucesso frente à avulsão dentária. Tanto o meio de estocagem quanto o tratamento de superfície dentária são fatores importantes para manter a viabilidade das células do ligamento periodontal ou reduzir os índices de acontecimentos da reabsorção radicular $^{16,17}$.

Com o desenvolvimento de avanços tecnológicos e tratamentos odontológicos têm aumentado suas taxas de sucesso ${ }^{18}$. Um desses avanços que tem se mostrado promissor é a aplicação da laserterapia de baixa potência (low level laser therapy - LLLT) com vistas ao auxílio do processo de reparo. A LLLT através da irradiação promove reações bioquímicas que podem alterar completamente o metabolismo celular (fotobiomodulação), resultando em estimulação do reparo tecidual e tem sido utilizado na odontologia em várias áreas devido seus benefícios antiálgico, anti-inflamatório e regenerativo ${ }^{19-21}$.

Dessa forma, o objetivo do presente trabalho foi de realizar uma revisão de literatura para compreender a ação do LLLT sobre as propedêuticas endodônticas e periodonticas; apresentar o conhecimento atual e científico sobre a eficácia da LLLT na avulsão dentária; e observar falhas de pesquisas sobre o tema.

ASSOCIAÇÃO DA LLT COM A ENDODONTIA

- Endodontia e Dor

Uma das complicações mais comuns no pós-operatório de procedimentos endodônticos é a dor $^{22}$. Ela possui caráter de intensidade leve à severa e pode ocorrer juntamente com a presença de edema ${ }^{22}$. A dor pode surgir em algumas horas ou dias após o tratamento ${ }^{22,23}$. Chen et al. ${ }^{23}$ realizaram uma revisão sistemática na qual avaliaram a efetividade do uso do LLLT na redução e analgesia da dor pós-operatória na terapia e retratamento do canal radicular. Ao comparar o LLLT com o um anti-inflamatório não esteroidal - ibuprofeno, foi constatado uma diminuição significativa da intensidade da dor quando utilizado o LLLT em relação ao ibuprofeno após a terapia do canal radicular. Além disso, a associação do LLLT e ibuprofeno no pré-operatório levou à uma maior redução de dor do que quando empregados sozinhos. Esse resultado corroborou com o realizado por Nabi e colaboradores ${ }^{24}$. Embora exista um amplo uso da farmacoterapia para amenizar a dor pósoperatória de procedimentos endodônticos, em razão de seus efeitos colaterais, há uma tendência da diminuição das aplicações de antiinflamatório não esteroidal e analgésicos e uma maior utilização do LLLT ${ }^{25}$.

- Cicatrização e Processo de Reparo

O LLLT produz efeitos anti-inflamatórios e analgésicos, aumentando a cicatrização e reparação dos tecidos no pós-operatório ${ }^{19,26}$. O LLLT promove a vasodilatação, aumentando o fluxo sanguíneo, a quantidade de oxigênio, migração de linfócitos e o relaxamento dos músculos lisos do endotélio, acelerando o processo de cicatrização de tecidos duros e moles $^{27}$. Metin et al. ${ }^{27}$ separaram setenta e seis casos de cirurgia endodôntica em incisivos superiores, dividindo-os em grupo controle (pacientes que não foram sujeitos a laserterapia) e grupo laser (pacientes submetidos ao laser imediatamente após a 
cirurgia e durante 7 dias no pós-operatório). Foi constatado que, no grupo laser, os pacientes apresentaram resultados mais satisfatórios com relação a cicatrização de feridas, área da lesão, índice periapical, volume da lesão e densidade óssea em relação ao grupo controle. Em contrapartida, Payer et al. ${ }^{28}$ dividiram setenta e dois casos de endocirurgia em incisivos e prémolares em três grupos, sendo um grupo de teste de LLLT (irradiação no intra e pósoperatório), um grupo de placebo (irradiação sem ativação do laser) e um grupo controle (sem utilização de LLLT e terapia placebo) e averiguaram não haver alteração significante em relação aos pacientes do grupo LLLT e do grupo placebo, porém, os pacientes do grupo controle obtiveram dores mais forte.

\section{- Pulpotomia}

A pulpotomia é o tratamento para a pulpite reversível e tem como finalidade a preservação dos dentes decíduos até o estágio da esfoliação ${ }^{29,30}$. Há muitas técnicas e materiais que podem ser utilizados na pulpotomia, especialmente relacionadas com o formocresol, hidróxido de cálcio, terapia a laser e entre outros ${ }^{29,31}$. A aplicação da laserterapia no tratamento da pulpotomia, de acordo com Marques et al. $^{31}$, tem capacidade de estimular o reparo e a cura dental, reduzir o edema e os efeitos inflamatórios e induzir a bioestimulação. No mais, o LLLT diminui o exsudato inflamatório, acelera a epitelização, propicia a vascularização e aumenta a produção de colágeno ${ }^{31}$. Além de tais benefícios, Ansari et al. $^{29}$ relataram melhora na calcificação, estimulação do tecido calcificado e biomodulação das células da polpa dentária com o uso do LLLT. Ao comparar o uso do laser de baixa potencia com os diferentes tipos de materiais existentes para o tratamento da pulpotomia, foi verificado que os grupos tratados com o LLLT não apresentaram reabsorção interna e o grupo da associação do LLLT com o hidróxido de cálcio apresentou menor grau de inflamação pulpar, sendo que 0 uso da laserterapia anterior ao uso do hidróxido de cálcio mostrou resultados mais satisfatórios. Em contrapartida, o formocresol obteve os maiores índices de reabsorção interna e estabeleceu respostas histológicas inadequadas na polpa radicular remanescente, como necrose e inflamação crônica no tecido conjuntivo.

ASSOCIAÇÃO DA LLT COM A PERIODONTIA

Os tratamentos periodontais têm como objetivo 0 controle de placa bacteriana, eliminação de bolsas profundas e de sangramento gengival ${ }^{32}$. Desse modo, a periodontia consiste na terapêutica de controle de inflamação e infecção, controle de fatores de risco e avaliação do periodonto de suporte ${ }^{32}$. O tratamento periodontal não cirúrgico é o mais efetivo para a eliminação das fontes de infecção, como a utilização do LLLT, que possui efeito anti-inflamatório, bioestimulativo e analgésico ${ }^{33}$. Ademais, Gündoğar et $\mathrm{al}^{34}$ constaram que o LLLT como complemento para o tratamento da periodontite crônica favorece em melhoras clínicas. Petrović et al. $^{35}$ demonstraram que o uso do LLLT reduz significativamente as bactérias da periodontite crônica e assim, a inflamação da gengiva. Segundo Lowe ${ }^{36}$, o laser retira cálculos, neutraliza a endotoxina, diminui a profundidade de bolsas e induz a uma bioestimulação, melhorando a cicatrização. Em vista disso, observou-se que o uso do laser de baixa potência pode ser utilizado como adjuvante na terapêutica periodontal.

ASSOCIAÇÃO DA LLT COM A AVULSÃO DENTÁRIA

A avulsão dentária é o deslocamento completo do dente da cavidade alveolar e tem como principal recurso terapêutico o reimplante dentário ${ }^{8,20}$. O reimplante dentário consiste no reposicionamento dos dentes em seus alvéolos, mas quando o reimplante não é imediato pode ocorrer a anquilose e a reabsorção dentária ${ }^{8,20}$. Santana et al. ${ }^{21}$ avaliaram o efeito da fototerapia a laser reparadora na polpa de dentes avulsionados e reimplantados de ratos e constatou-se um aumento na espessura da camada de dentina reacional e escassez de inflamação. Carvalho et al. ${ }^{20}$ analisaram os incisivos superiores direitos extraídos de ratos e que foram submetidos a irradiação a laser antes do reimplante tanto na superfície radicular e quanto no alvéolo e observaram um efeito de biomodulação dos dentes reimplantados dos ratos. De igual modo, Carroll et al. ${ }^{19}$ verificaram que o LLLT melhora a cicatrização, reduz a inflamação e controla a dor.

CONSIDERAÇÕES FINAIS

A aplicação do LLLT na endodontia, periodontia e no tratamento da avulsão dentária é prerrogativa devido aos efeitos positivos de seu uso. Na endodontia, o seu uso suscita uma diminuição dos sintomas do pós-operatório, como a mitigação da dor significativamente maior em relação aos anti-inflamatórios e redução do edema; além disso, propicia o aumento da cicatrização, estimulação do reparo e induz a bioestimulação das células da polpa dentária. $\mathrm{Na}$ periodontia, além dos fatores já citados, há uma diminuição das bactérias que induzem a periodontite crônica e inflamação da gengiva. Após o reimplante dentário em razão da avulsão do elemento dental, o uso do LLLT 
evita uma possível reabsorção radicular, aumenta a espessura da camada de dentina e diminui a sintomatologia da inflamação. A irradiação do LLLT promove reações bioquímicas, ocorrendo a fotobiomodulação (alteração completa do metabolismo celular) que origina a estimulação do reparo tecidual e regeneração. Ademais, diminui 0 processo inflamatório, impede a ocorrência de reabsorção radicular inflamatória externa e anquilose nos procedimentos de reimplante do elemento dental devido a avulsão. Apesar dos benefícios e dos seus efeitos já relatados na literatura, ainda faltam estudos sobre a aplicação do LLLT, principalmente aos procedimentos realizados em decorrência de uma avulsão dentária. Os estudos e os tratamentos são promissores e apontam para uma perspectiva de tratamento eficaz, com os menores sintomas no pós operatório.

\section{REFERÊNCIAS}

1. Diangelis AJ, Andreasen JO, Ebeleseder KA, Kenny DJ, Trope M, Sigurdsson $A$ et al. International Association of Dental Traumatology guidelines for the management of traumatic dental injuries: 1. Fractures and luxations of permanent teeth. Dent Traumatol. 2012;28(1):2-12.

2. Cantaloube D, Daupleix C, Chikhani L, Caucanas D. Sequelae of Dental Trauma: Forensic Medical Aspects. Rev Stomatol Chir Maxillofac. 2006;107(4):294-302.

3. Zaleckiene V, Peciuliene V, Brukiene V, Drukteinis S. Traumatic Dental Injuries: Etiology, Prevalence and Possible Outcomes. Stomatologija. 2014;16(1):7-14.

4. Lam R, Abbott P, Lloyd C, Lloyd C, Kruger E, Tennant M. Dental Trauma in an Australian Rural Centre Dent Traumatol. 2008;24(6): 663-70.

5. Díaz JA, Bustos L, Brandt AC, Fernández BE. Dental injuries among children and adolescents aged 1-15 years attending to public hospital in Temuco, Chile. Dent Traumatol. 2010;26(3): 254-61.

6. Eyuboglu O, Yilmaz Y, Zehir C, Sahin H. A 6year investigation into types of dental trauma treated in a paediatric dentistry clinic in eastern Anatolia region, Turkey. Dent Traumatol. 2009;25(1):110-14.

7. Andersson $L$, Andreasen JO, Day $P$, Heithersay G, Trope M, DiAngelis AJ et al. Guidelines for the Management of Traumatic Dental Injuries: 2. Avulsion of Permanent Teeth. Pediatr Dent. 2017;39(6):412-19.

8. Longo DL, Fumes AC, Küchler EC, Paula-Silva FWG, Nelson-Filho P, Silva LAB. Efficiency of different storage media for avulsed teeth in animal models: a systematic review. Dent Traumatol. 2018;34(1):12-19.

9. Chauhan R, Rasaratnam L, Alani A, Djemal S. Adult dental trauma: what should the dental practitioner know? Prim Dent J. 2016;5(3): 70-81.

10. Moran I, James M, Cook W, Perry M. Tooth avulsion. BMJ. 2016;353:i1394.

11. Amaral MF, Almeida MM, Faria LP, Brandini DA, Poi WR, Okamoto R. Treatment of extrusive luxation in permanent teeth: literature review with systematic criteria. J Contemp Dent Pract. 2017;18(3):241-45.

12. Trope M. Protocol for treating the avulsed tooth. W V Dent J. 1996;70(2):19-23.

13. Hammarsröm L, Pierce A, Blomlöf L, Feiglin B, Lindskog $S$. Tooth avulsion and replantation--a review. Endod Dent Traumatol. 1986;2(1):1-8.

14. Christophersen P, Freund M, Harild L. Avulsion of primary teeth and sequelae on the permanent successors. Dent Traumatol. 2005; 21(6):320-23.

15. Giannetti L, Murri A, Vecci F, Gatto R. Dental Avulsion: Therapeutic protocols and oral healthrelated quality of life. Eur $\mathrm{J}$ Paediatr Dent. 2007;8(2):69-75.

16. Poi WR. Effect of low-level laser therapy on the healing process after tooth replantation: a histomorphometrical and immunohistochemical analysis. Dent Traumatol. 2011;27(1):30-9.

17. Andersson L, Andreasen JO, Day P, Heithersay G, Trope M, Diangelis AJ et al. International Association of Dental Traumatology guidelines for the management of traumatic dental injuries: 2. Avulsion of permanent teeth. Dent Traumatol. 2012;28(2):88-96.

18. Mortman RE. Technologic advances in endodontics. Dent Clin North Am. 2011;55(3): 461-80.

19. Carroll JD, Milward MR, Cooper PR, Hadis M, Palin WM. Developments in low level light therapy (LLLT) for dentistry. Dent Mater.2014; 30(5):465-75.

20. de Carvalho FB, Andrade AS, Barbosa AF, Aguiar MC, Cangussu MC, Pinheiro AL et al. Evaluation of laser phototherapy $(\lambda 780 \mathrm{~nm})$ after dental replantation in rats. Dent Traumatol. 2016;32(6):488-94.

21. de Santana DA, Fonseca GF, Ramalho LMP, Rodriguez TT, Aguiar MC. Effect of low-level laser therapy $(\lambda 780 \mathrm{~nm})$ on the mechanically damaged dentin-pulp complex in a model of extrusive luxation in rat incisors. Lasers Med Sci. 201732(9):1995-2004.

22. Sathorn C, Parashos P, Messer $H$. The prevalence of postoperative pain and flare-up in single- and multiple-visit endodontic treatment: a systematic review. Int Endod J. 2008;41(2):91-9. 
23. Chen Y, Chen XL, Zou XL, Chen SZ, Zou J, Wang $Y$. Efficacy of low-level laser therapy in pain management after root canal treatment or retreatment: a systematic review. Lasers Med Sci. 2019;34(7):1305-1316.

24. Nabi S, Amin K, Masoodi A, Farooq R, Purra AR, Ahangar FA. Effect of preoperative ibuprofen in controlling postendodontic pain with and without low-level laser therapy in single visit endodontics: A randomized clinical study. Indian J Dent Res. 2018;29(1):46-50.

25. Asnaashari $M$, Ashraf $H$, Daghayeghi $A H$, Mojahedi SM, Azari-Marhabi S. Management of post endodontic retreatment pain with low level laser therapy. J Lasers Med Sci. 2017;8(3): 128-31.

26. Saito CT, Gulinelli JL, Panzarini SR, Garcia VG, Okamoto R, Okamoto T et al. Effect of low-level laser therapy on the healing process after tooth replantation: a histomorphometrical and immunohistochemical analysis. Dent Traumatol. 2011;27(1):30-9.

27. Metin R, Tatli U, Evlice B. Effects of low-level laser therapy on soft and hard tissue healing after endodontic surgery. Lasers Med Sci. 2018;33(8):1699-706.

28. Payer $M$, Jakse $N$, Pertl $C$, Truschnegg $A$, Lechner E, Eskici A. The clinical effect of LLLT in endodontic surgery: a prospective study on 72 cases. Oral Surg Oral Med Oral Pathol Oral Radiol Endod. 2005;100(3):375-79.

29. Ansari G, Safi Aghdam H, Taheri P, Ghazizadeh Ahsaie M. Laser pulpotomy-an effective alternative to conventional techniquesa systematic review of literature and metaanalysis. Lasers Med Sci. 2018;33(8):1621-629.

30. Ansari G, Morovati SP, Asgary S. Evaluation of Four Pulpotomy Techniques in Primary Molars: A Randomized Controlled Trial. Iran Endod J. 2018;13(1):7-12.

31. Marques NC, Neto NL, Rodini Cde O, Fernandes AP, Sakai VT, Machado MA et al. Low-level laser therapy as an alternative for pulpotomy in human primary teeth. Lasers Med Sci. $2015 ; 30(7): 1815-22$.

32. Bartold PM. Lifestyle and periodontitis: The emergence of personalized periodontics. Periodontol 2000. 2018;78(1):7-11.

33. Ren C, McGrath C, Jin L, Zhang C, Yang Y. The effectiveness of low-level laser therapy as an adjunct to non-surgical periodontal treatment: a meta-analysis. J Periodontal Res. 2017;52(1):8-20.

34. Gündoğar H, Şenyurt SZ, Erciyas K, Yalım M, Üstün K. The effect of low-level laser therapy on non-surgical periodontal treatment: a randomized controlled, single-blind, split-mouth clinical trial. Lasers Med Sci. 2016;31(9): 1767-73.
35. Petrović MS, Kannosh IY, Milašin JM, Mihailović DS, Obradović RR, Bubanj SR et al. Clinical, microbiological and cytomorphometric evaluation of low-level laser therapy as an adjunct to periodontal therapy in patients with chronic periodontitis. Int $J$ Dent Hyg. 2018;16(2):e120-27.

36. Low SB, Mott A. Laser technology to manage periodontal disease: a valid concept? J Evid Based Dent Pract. 2014;14(Suppl):154-59.

\section{CONFLITO DE INTERESSES}

Os autores declaram não haver conflitos de interesse

\section{AUTOR PARA CORRESPONDÊNCIA}

\author{
Christine Men Martins \\ Curso de Odontologia, \\ Universidade do Oeste Paulista (UNOESTE), \\ Rua José Bongiovani, 700 \\ 19050-920, Presidente Prudente, Sao Paulo, Brazil. \\ Tel.: +55 (18) $3229-2003$, \\ e-mail: christinemen@hotmail.com
}

Submetido em 03/06/2020

Aceito em 23/10/2020 